\title{
FGEHF: Authenticated Web Based Application for Human Resource Management System
}

\author{
Sijjad Ali khuhro ${ }^{1 *}$, Muhammad Aamir Panhwar ${ }^{2}$, Attaullah Buriro', \\ Adeel Ahmed', Suhail Ahmed Khuhawar ${ }^{3}$ and Shoukat Hayat ${ }^{3}$ \\ 'University of Science and Technology of China, Anhui, China; \\ sijjadali786@mail.ustc.edu.cn, adeelahmed@mail.ustc.edu.cn \\ 2Beijing University of Posts and Telecommunications, China; maamirpanhwar@hotmail.com \\ ${ }^{3}$ Quaid-i-Azam University, Islamabad, Pakistan; qausuhail@gmail.com, shoukathayat444@gmail.com \\ ${ }^{4}$ Khwaja Fareed University of Engineering and IT, Rahim Yar Khan, Pakistan; attaullah.buriro@kfueit.edu.pk
}

\begin{abstract}
Objectives: HRM systems have faced a lot of challenges in recent years because their working is dependent on manual/ files system. Manual management of an organization and its employees' information along with their payroll processing, file management, report generation and leave management is really time-consuming. Data may also involve human errors which increases the risk factor. These kinds of problems rise in all kind of organizations, especially in the absence of Electronic Web-based system. Methods/Statistical Analysis: Our proposed method -Authenticated web-based application for the human resources management system is beneficial for Federal Government Employee Housing Foundation (FGEHF), because this organization is working manually since 1988. It makes difficult for the HR to manage all those data records without any system, so we have tried to solve this problem by proposing a web-based state-of-the-art solution. Findings: The underlying approach of our work is to facilitate the staff of FGEHF, by delivering an automatic system, to make their working easier and effective. Additionally, the utilization of the proposed HRM system could save time, cost and easier maintenance of error-free data. All registered users and their completed record are maintained on the database server in a secure manner. All data is promised to be secure and would not be possible for an intruder to access the private information of others employees. Application/Improvements: The efficiency of the proposed technique is validatedByASP.Net and C\#.NET is used as programming languages for developing HRM web-based system.
\end{abstract}

Keywords: Authentication, Database, Management, ASP.NeT, C\#.NeT, HRMS

\section{Introduction}

A Human Resource Management system-HRMS or Human Resource Information System - HRIS, deals with the systems and methods involving HRMS and the Information Technology (IT) altogether. More specifically, it combines HRMS as a discipline for fundamental activities and its progression in the area of IT. However, in data processing systems the main purpose of programming carries out uniform procedures and packages of Enterprise Resource Planning (ERP) software. In the regard of ERP schemes, they have their foundations for software and could assimilate information over multiple applications in one unique or universal database. The association among them, in the sense of financial as well as human resources components, undergoes in one unique database that is a very important difference to the individual and exclusive growth precursors, they make this kind of software application both rigid and flexible. All web application can be accessed on computers, mobile phones, and many users avail of this kind of facility in Pakistan ${ }^{1}$. 
The purpose of HRMS organizations is mainly the administration and is common in each organization. These organizations are ranked based on the transparency in selection, evaluation and workforce recruitment and management procedures. Additionally, the effective organization and real management of employees requires easier and multifaceted procedures. The main function of HRMS based on tracking out the employees' information which could include personal data, history, skills, experience, reward, and salaries. To decrease the cumbersome manual work and wastage of time, organizations have already been moving to the electronic computerized world using specialized and customized HRMS solutions. So the smooth operating of those HRMS solutions require HR personnel with the specialization in IT, to grow and handle, effectively the HRMS ${ }^{2}$.

We propose a unique and different methodology for overcoming the problem of existing schemes. Our solution is a web-based application for FGEHF, which agrees with the interaction for each employee relating to their organization. Our application helps the HR of any organization to apprise employee history whenever there happens a change in the employees' profile.

The rest of paperis organized as below:Section 2 explain some necessary background. Section3 exlpains the problem and the mission statement. Section 4, expalins the scope of our work. Section 5 , discusses the security solution of the proposed web service. Section 6, discusses the system design and Section 8, explains the system's structure. Section 9, concludes the paper and the related possible future work.

\section{Background Study About FGEHF}

The federal government of Pakistan took a major initiative to offer accommodation to its employees on the self-financing scheme on possession basis in the capital city - Islamabad, Pakistan. The scheme received a marvelous response from the all the stakeholders including the employees and the involved government subsidiary organization.

Later, in 1992, another phase of the said scheme was launched, keeping in view the severe shortage of housing in the capital of Pakistan. The same scheme was also launched in other major cities of Pakistan, such as Karachi, and Peshawar, to overcome the shortage of houses and rising demands of federal government employees.

FGEHF was founded in March 1990, and it got registered as a limited company according to the companies law 1984. Basically, it was entrusted with the regard of a job for deployment of self-financing housing schemes on the basis of federal government employee ownership. They built 16000 houses in the duration of 5 years with additional 4000 residential plots distributed among the applicants based on the lucky draw to build the houses by themselves.

The main issue the employees of FGEHF faced was the manual handling of personal data, which made the management very difficult and cumbersome for the HR department. We proposed a computerized and online system which helps HR professional to conveniently and efficiently handle the employee's data. Our proposed system is meant to save the time and cost which may incur in the manual workflow. Intuition assessment proves our system very secure, flexible and easy to use.

\section{Mission Statement}

The goal of our project is to create a unified IT environment for FGEHF employees. Our system can meet the following responsibilities:

- Employees Information and their management.

- Organization Information and its management.

- Employee Payroll Information and its management.

- Files tracking and their management.

- Users and their role management.

The HRMS includes a comprehensive employee information database comprised of employee biodata, information about work, recipient information, and other relevant information for every employee. It is a database for employee self-service access module allowing employees to update their personal information and query for any information.

\subsection{Features of Proposed System}

- All the work is done automatically hence there is no need of extra manual effort.

- It makes easy and saves time in finding an employee's related data. 
- It is very fast and accurate system.

- It is secure system and no danger of losing the employees data in any case.

- Security of data information from attacking.

\subsection{Problem Description in FGEHF}

FGEHF currently has 340 employees in Islamabad office. Manual management of an organization and its employees' information along with their payroll processing, file management, report generation and leave management is cumbersome and time-consuming. Data may also involve human errors which increases the risk factor. Currently, there is no automatic system available in FGEHF, which provides efficient management of all problems related to the management of the organization and its employees.

\section{Project Scope}

The objective of our proposed system is to facilitate the organization in the following ways:

- Organization Management.

- Employee Management.

- Pay-Roll management.

- Office Files Tracking.

- User and Role Management.

- Reports of all above Modules.

\subsection{Organization Management}

This module contains information about Federal Government Employee Housing Foundation (FGEHF). This information includes how many wings are there and also describes sections in each wing along with their abbreviations. It also contains a complete record of all wings and the information in the sections. This module has the capability to search about wings, section details and have complete user/role management.

\subsection{Employee Management}

This module contains information about all employees of FGEHF. It has the functionalities to add, delete, update and search about following entities:

- Employee personal record.

- Official record.

- Status.
- Qualification.

- Domicile.

- Religion.

- Bank detail.

- Cities.

\subsection{Pay-Roll Management}

The payroll Management component mechanizes the payroll procedure by collecting employee's information. Calculate numerous inferences and allowances, advances and making intermittent pay slips as well as employee reports. Payroll Management system is used to provide an option to generate the salary automatically every month.

This module contains information about Employee's payroll of the FGEHF. It allows to Add, Update, Search, and user/roll management about following entities:

- Payroll generation.

- Employee Allowances.

- Deductions.

- Pay slips.

- Advances.

- BPS.

- Pay Types.

- Reporting.

\subsection{Office Files Tracking}

This module contains information about office files. Their complete track is stored in data base, their current location, where they come from and where these files will be stored. This module help in searching any file efficiently and accurately. It has the following functionalities:

- Add File.

- Update File.

- Update File

- Search File.

- Import file

- Export file

\subsection{User and Roll Management}

This module contains information about user and his/ her roll and manages that information. Deferent users are given different login ID and password and administrator can assign specific role to any user. User can access only that function and can perform those task which are permitted by administrator. 


\subsection{Reports Generation}

This module is meant to generate reports for the HR department, on the basis of their requirement. Our module can generate the following types of reports:

- Organization/wings/sections reports.

- Employees Report.

- Employee payroll reports.

- Files reports.

\section{Security Solutions in Web Service}

In our security analysis, we tried to explore and report below some security issues in web-services against the adversaries. Interested readers are referred to Figure 1 to better understand our proposed scheme better.

\subsection{Apparatus Deployment}

One of the fundamental things is to secure data against the adversaries by using the dedicated hardware in an intelligent way. As such, we put our database behind the firewall.

\subsection{User Authentication}

The access to the web service should be restricted to the legitimate user only. Thus, each access should be authenticated. This would help the responsible to detect the malicious attempts while they provide wrong information.

\subsection{Safeguarding Data}

It is the responsibility of the corresponding manager to make sure the security of the collected data. Managers need to ensure the controlled list of files and SQL servers. Our proposed scheme identifies the users (authenticates) before they could access the web system. In this way, our scheme ensures data integrity.

\subsection{Track User Activity}

The web-system needs to monitor the user activities, in real time, in order to avoid any possible non-repudiation. The manager responsible should be able to track the user's activities while accessing the system.

\section{System Design}

System design plays very important part in the system functionality. Researchers should focus on design flow

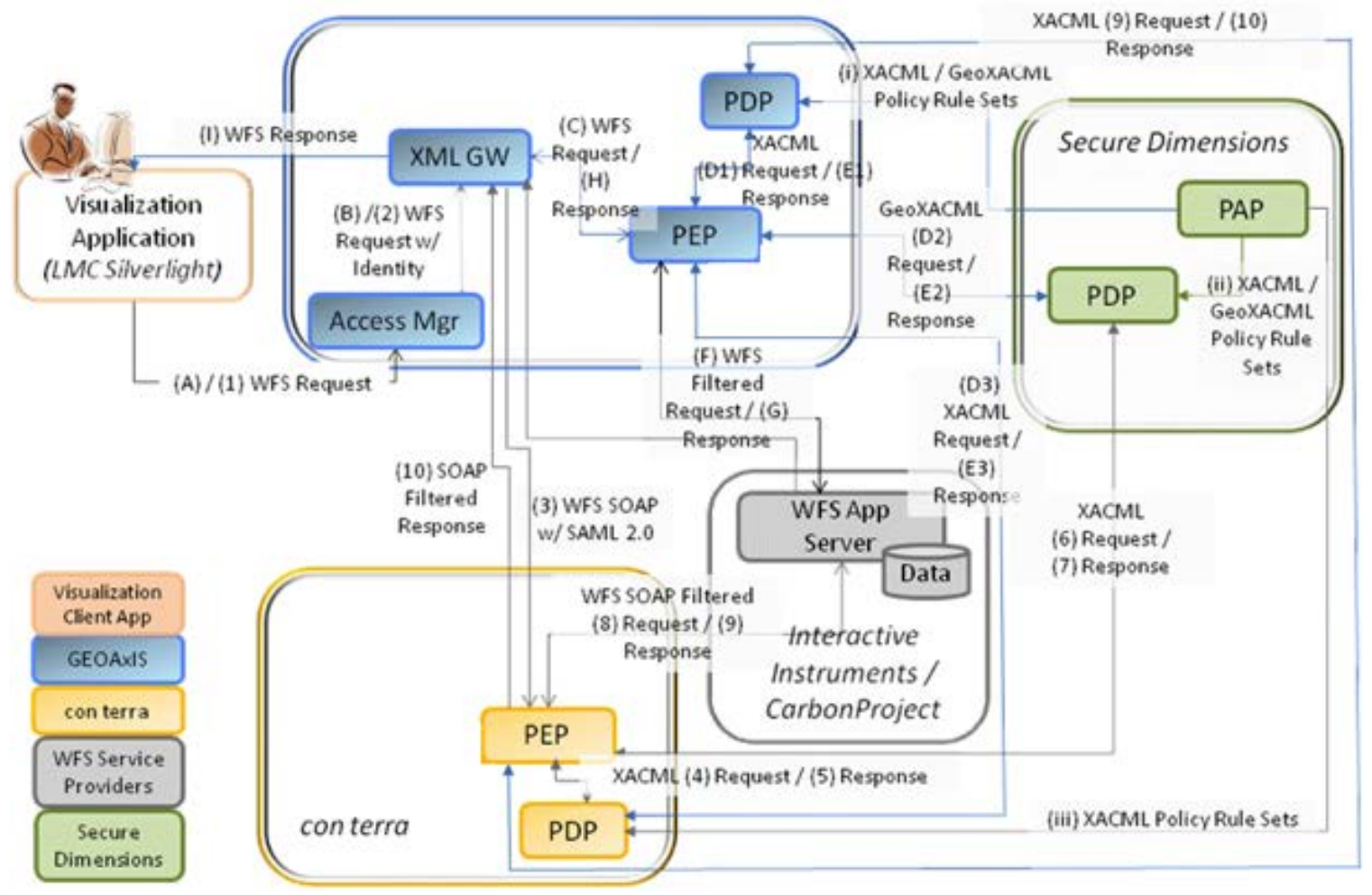

Figure 1. Mechanism for user authentication. 
and cost risk, time, etc., before designing any such system.

\subsection{System Architecture}

In this diagram our proposed system shows the relationship between each component within the system. This diagram is readable and is easy to understand for readers as shown in Figure 2.

\subsection{Class Diagram}

We illustrate the design of our system using system classes (shown in Figure 3). A class describes the properties and behavior of the object. A class diagram describes the view in expressions of classes and relationship among the class'sentities ${ }^{3-5}$.

\section{System Implementation}

We start the software development phase after the completion of system design. Main goal of this implementation is making the system ready for execution ${ }^{6}$. Additionally, the purpose is to understand the application. The system includes the performance of each hardware component, software usage, and any other added tool used for the development and the possible problems occurred in the installation phase. We implemented the system with the hope that if would be working correctly, efficiently, and quickly.

\subsection{Programming Languages Selection}

For the development of the "FGEFH" HRMS, ASP.Net and C\#.NET are used as programming languages.

\subsection{Development Tools}

Developments tools used to implement the system are:

- Microsoft Visual Studio 2010: This is main tool for coding in C\#.NET.

- Microsoft SQL server 2008 Management studio: Purpose of this software to handle the database of the system.

- Microsoft word 2007: The advantage of this software to handle for documentation purposes.

- Microsoft Visio: This tool is used for the drawing UML diagrams.

- Microsoft Excel: Can use to import data to store in database, and export data as report.

\subsection{Code Brief in C\#}

We show the implemented business logic of organization, wings, and section in the code snippet shown in Figure

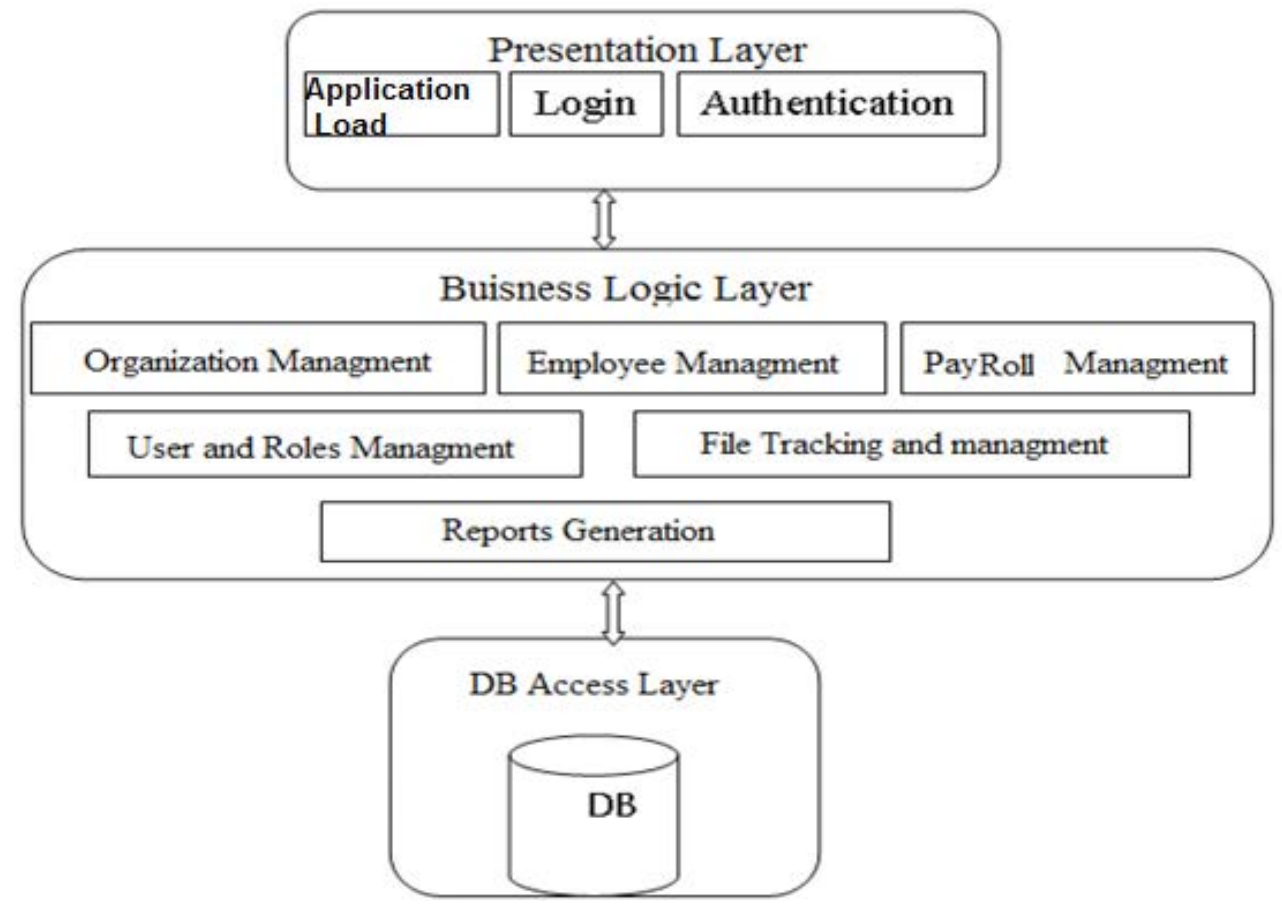

Figure 2. Architecture diagram. 


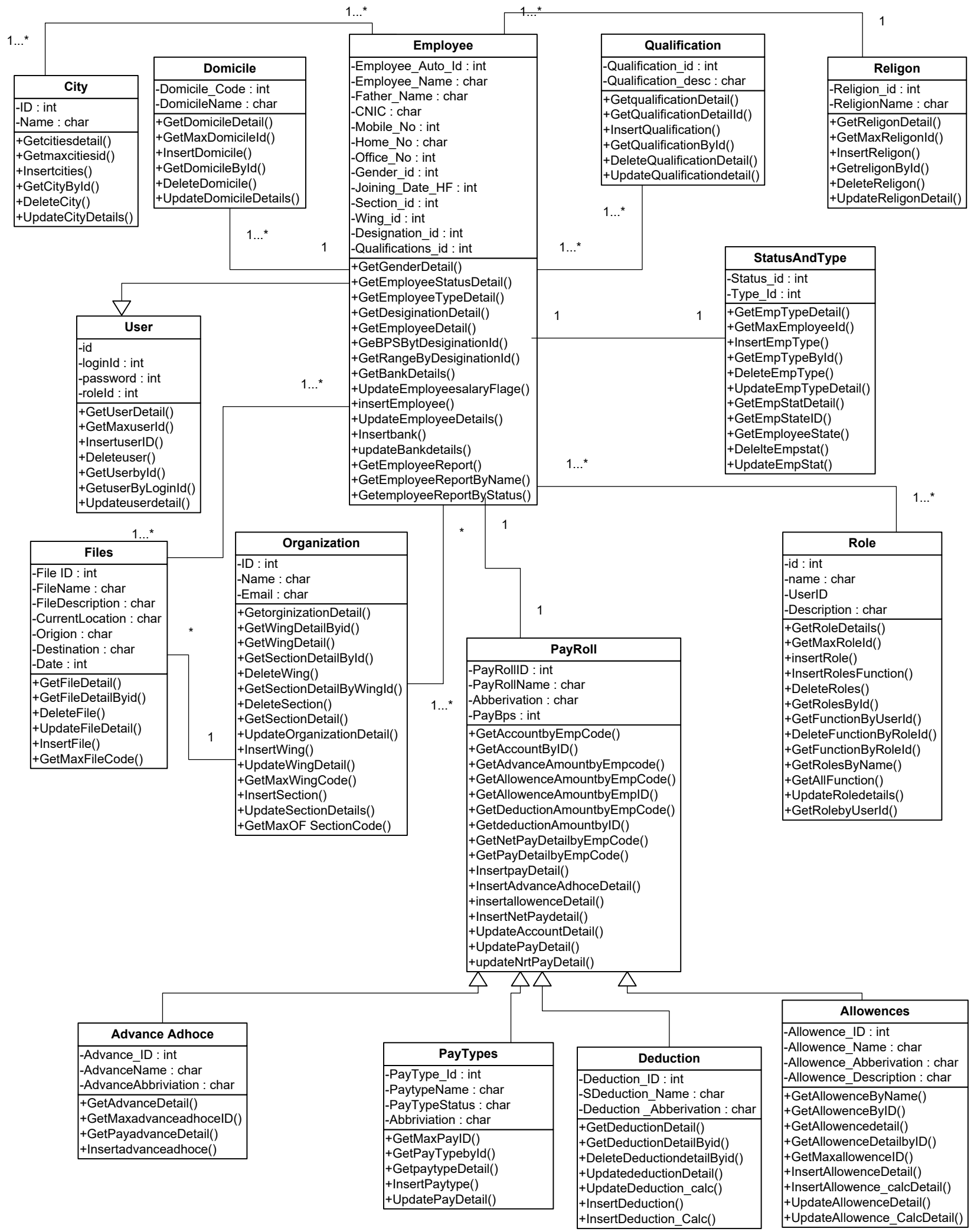

Figure 3. HRMS class diagram. 
4. It shows that different queries can be implemented to store and retrieve data into/from the database.

The code snippet (Figure 5) contains the business logic implemented for users, queries, and to store user related data into the database and later for its retrieval.

Additionally, thecode shown in Figure 5, shows the class implemented and connected with the database via connection string. Screen shot database class is imple- mented and application is connected with database via connection string.

\section{System View Structure}

Some screen shots of the system are displayed here which will help the readers to better understand the use of the system, according to their desired actions?

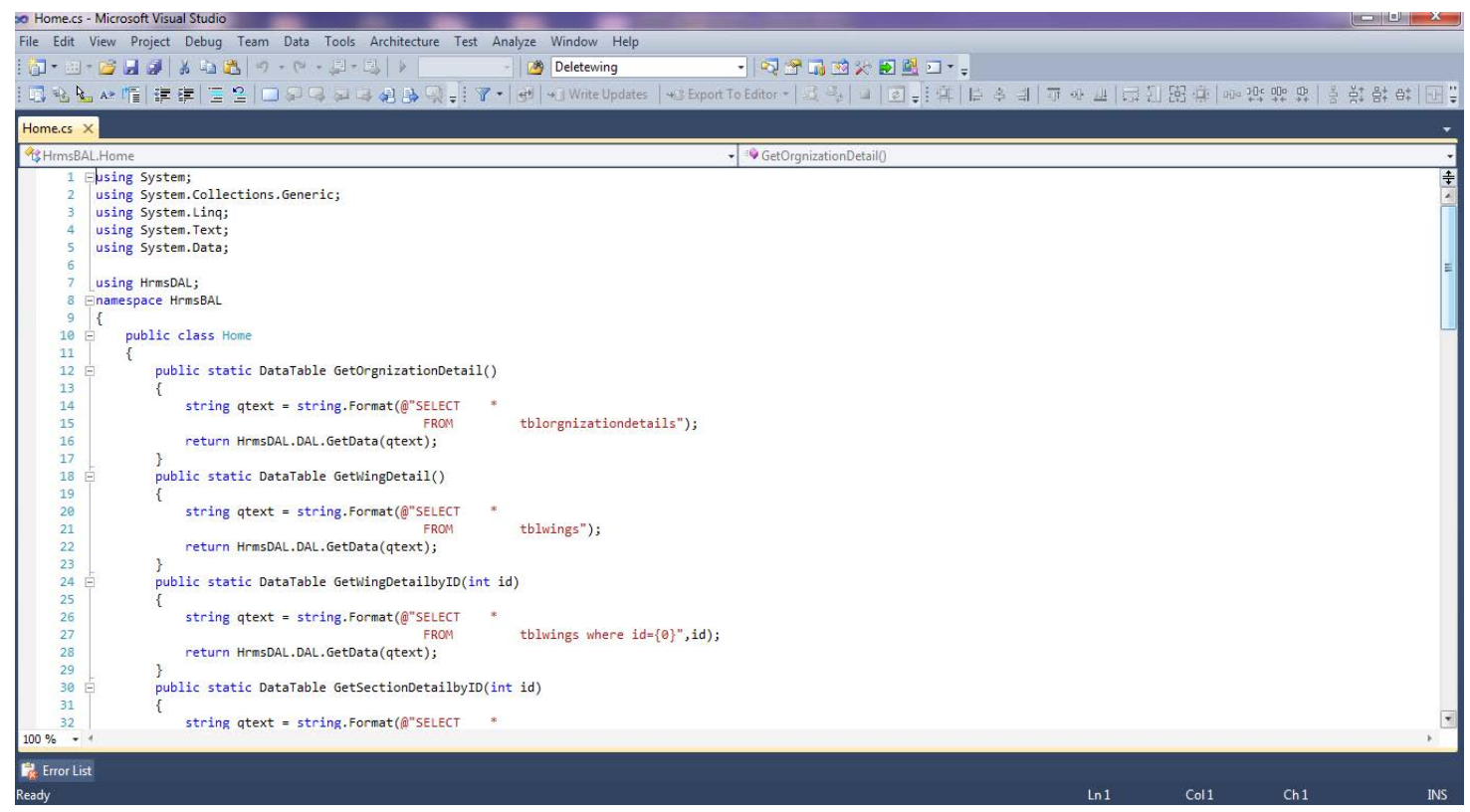

Figure 4. User database.

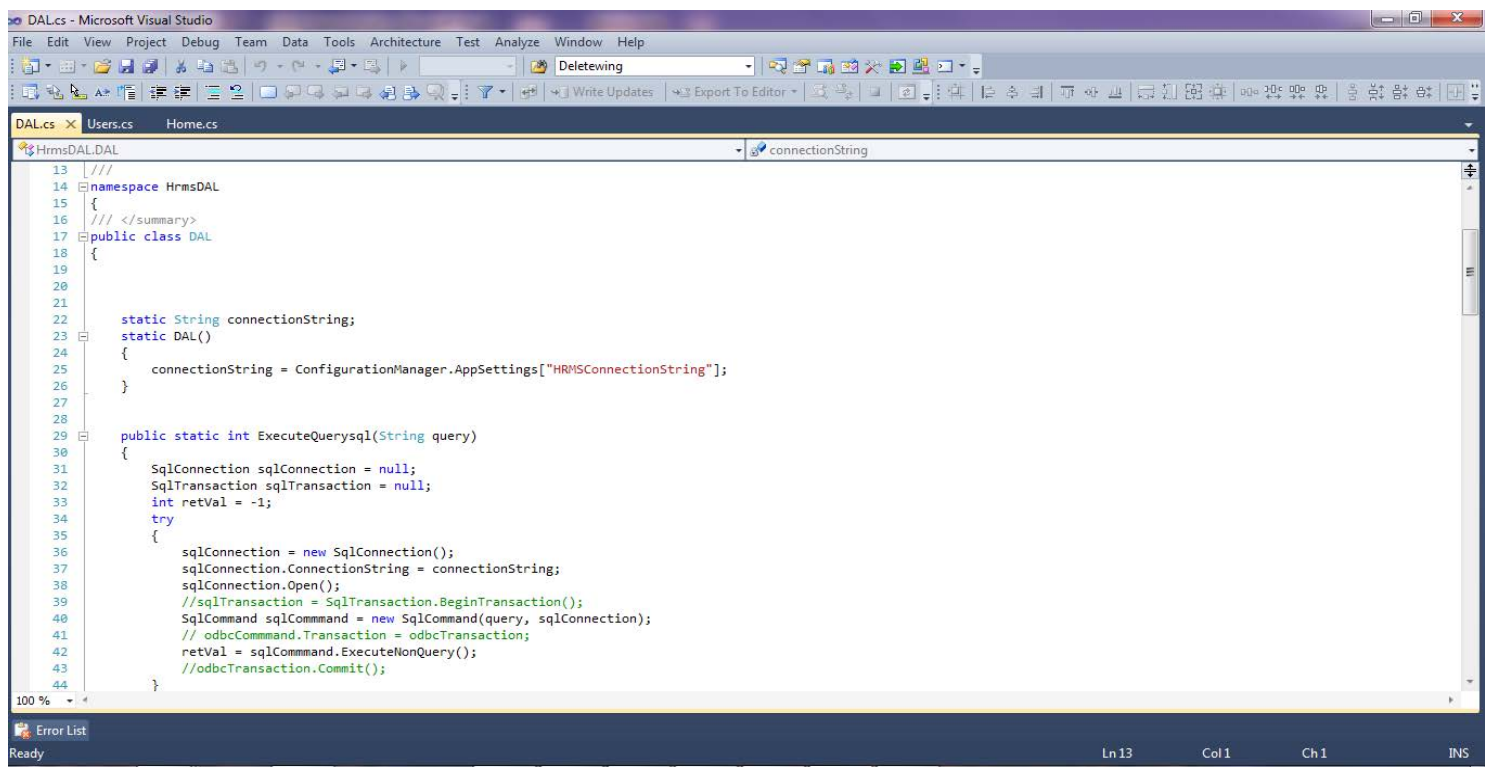

Figure 5. Connction between database vs strings. 


\subsection{Login Page}

The user can access the resources of the system upon successful authentication by providing their username and password. This login page is depicted in Figure 6.

\subsection{Home Page}

User can access those functionalities for which he/she is assigned the role by the admin. User can navigate through top menu or left side menu (Figure 7).

\subsection{Add Wing Page}

Users can enter the new wing information by filling up the required fields. The "Save" button saves the entered record in the database while "Cancel" button cancels the entered information. The process is illustrated in Figure 8.

\subsection{View section details Page}

User can visit this page by clicking on sections link. Here the users can edit and delete the section by clicking Edit section
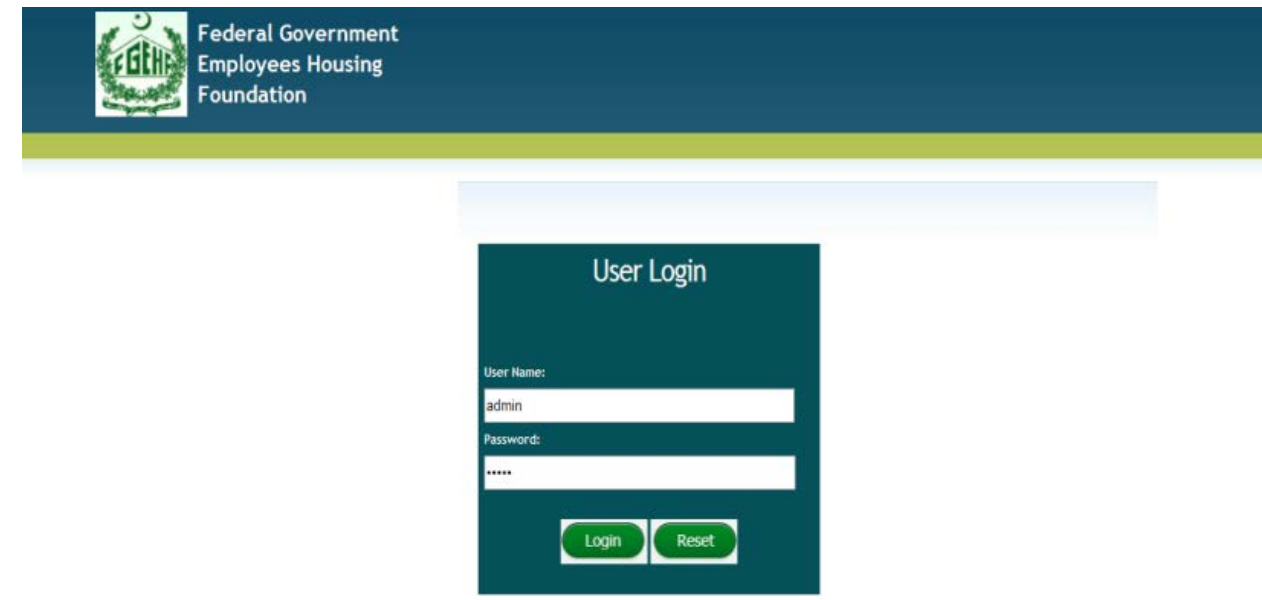

Figure 6. Login page.

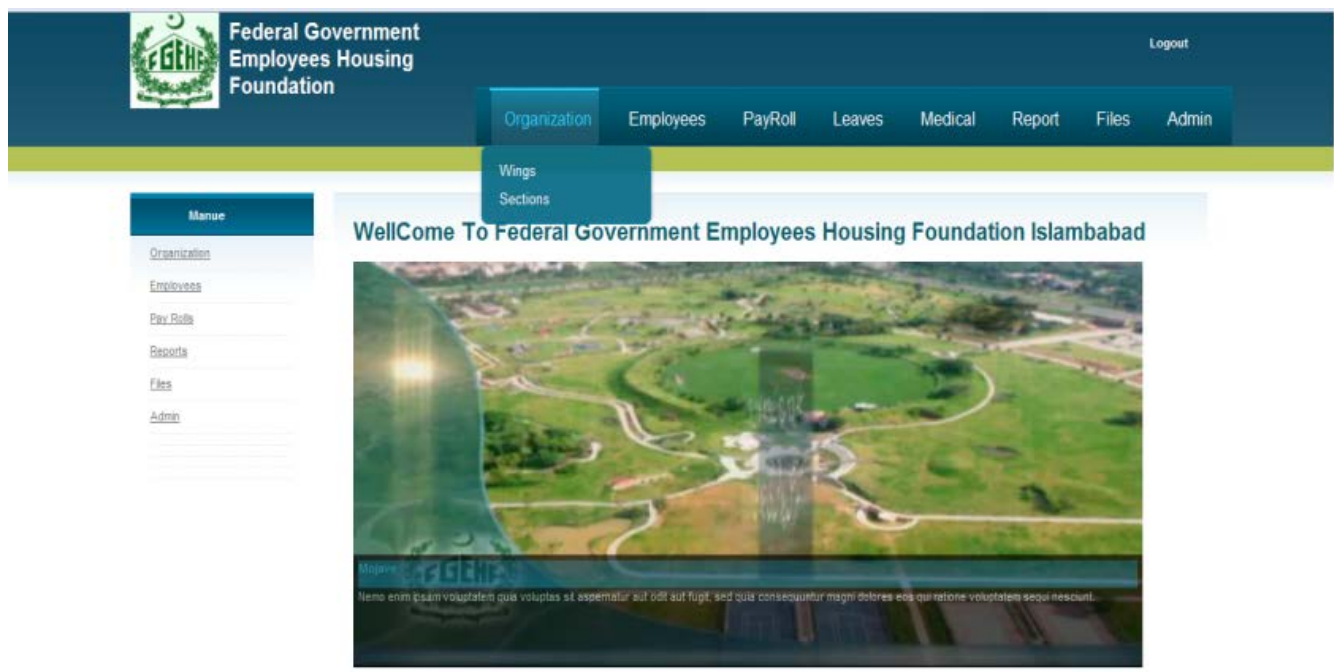

Figure 7. Home page. 


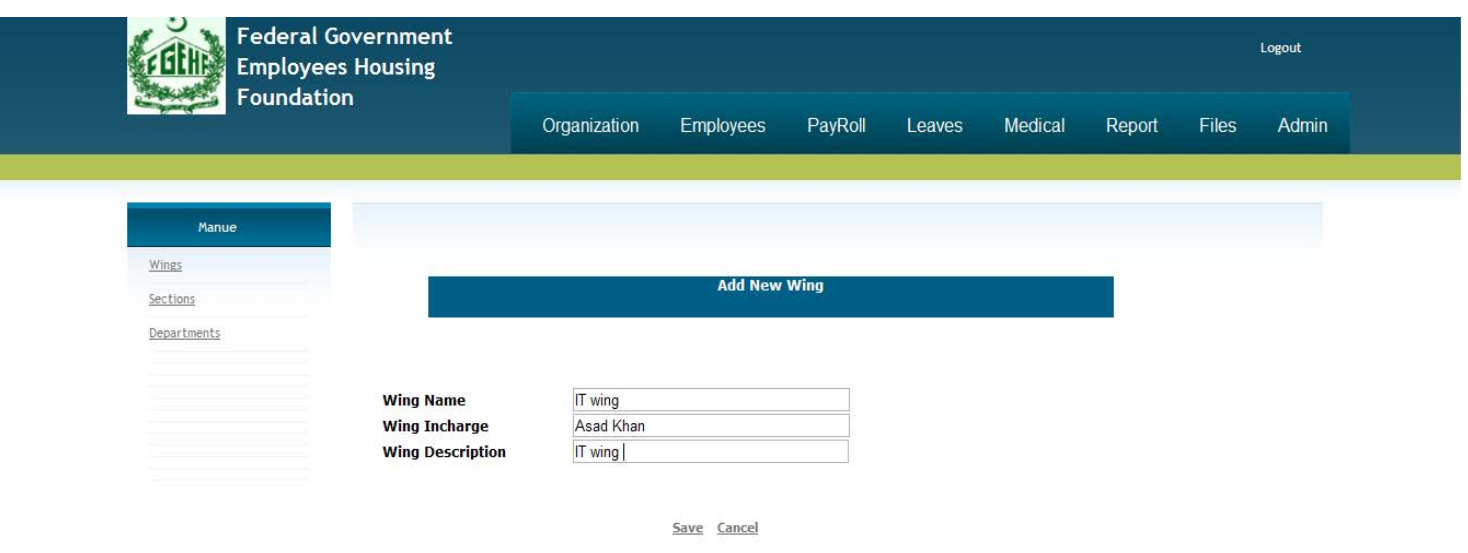

Figure 8. Wings page.

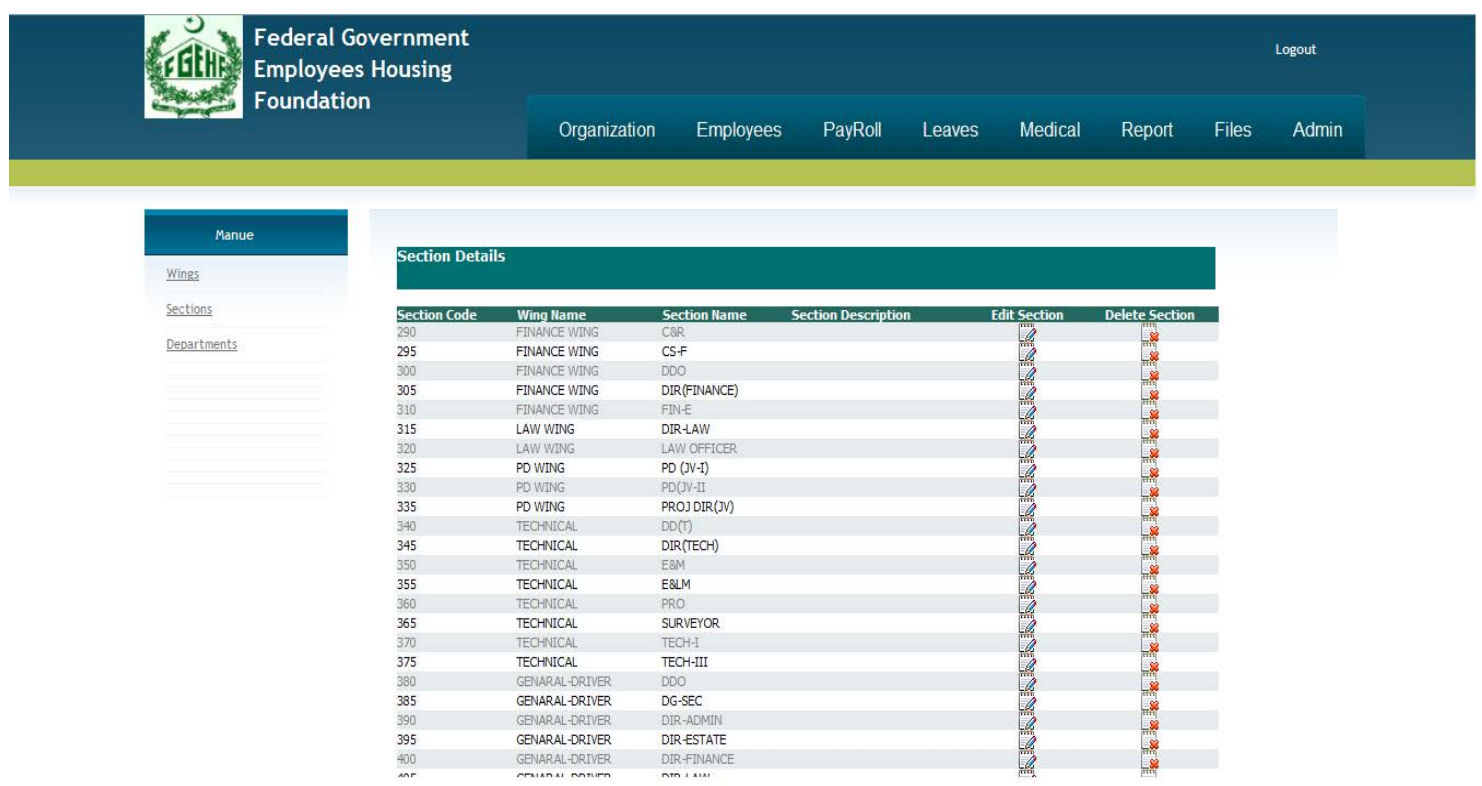

Figure 9. Section detail page.

and Delete section link, respectively, but only those users who are assigned the role to perform these task (Figure 9).

\subsection{Add Employee Page}

In this page (Figure 10) the user can add new employee information by filling up the required fields.

\subsection{Search Employee Page}

User can search specific employee's record by entering Employee's code or name or their CNIC \# when user clicks on respective search button employee's details will display on screen (Figure 11). 


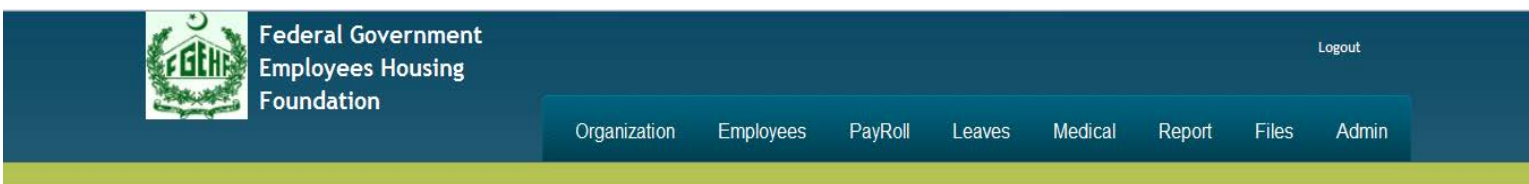

\begin{tabular}{l|}
\hline Manue \\
\hline Search Emplovee \\
Qualification \\
Domicle \\
Religion \\
City \\
StatuseTrpe
\end{tabular}

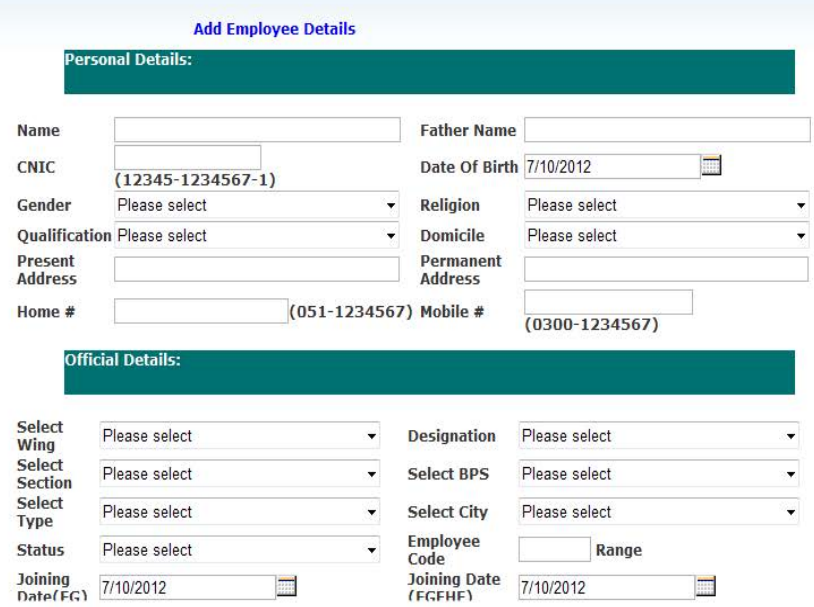

Figure 10. Employees page.

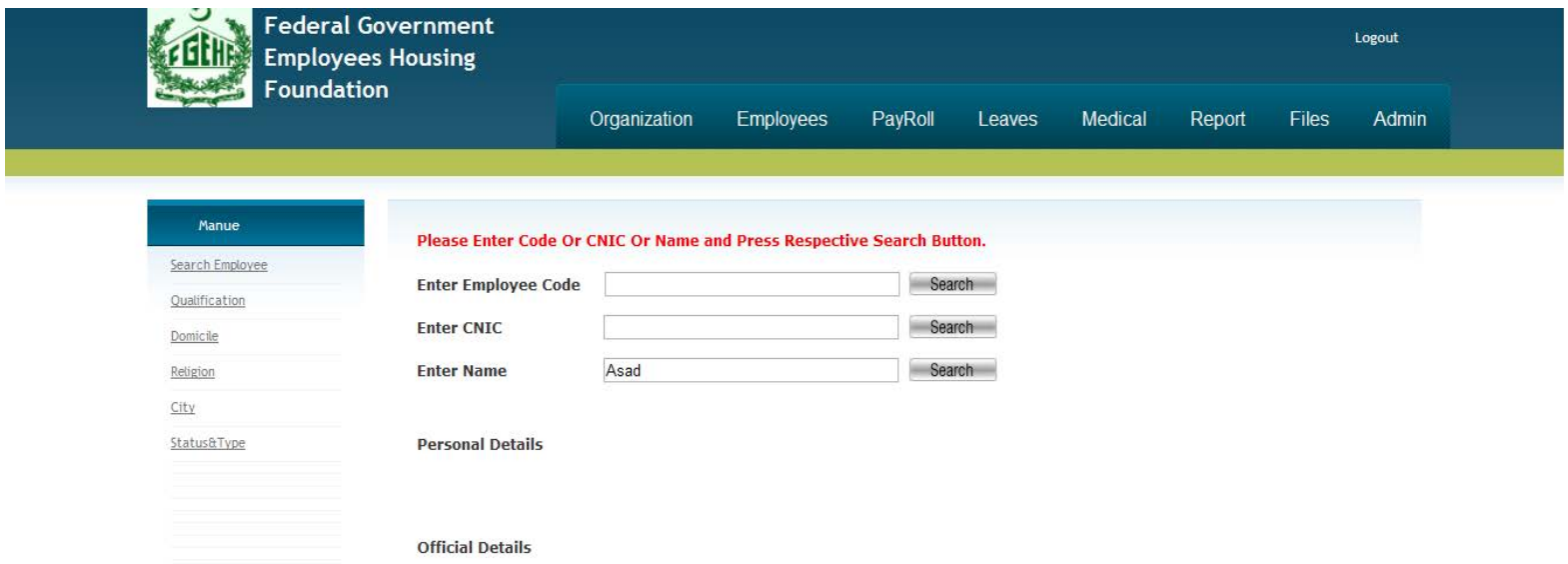

Figure 11. Employee search page.

\subsection{Add new file Page}

User can add new file record by filling up all the required fields and can save the entered information in database by clicking on save link (Figure 12).

\subsection{View File Page}

When user clicks on File Tab, this page appears which displays all the entered file records. Authenticated users would be able to perform the addition and deletion. The 

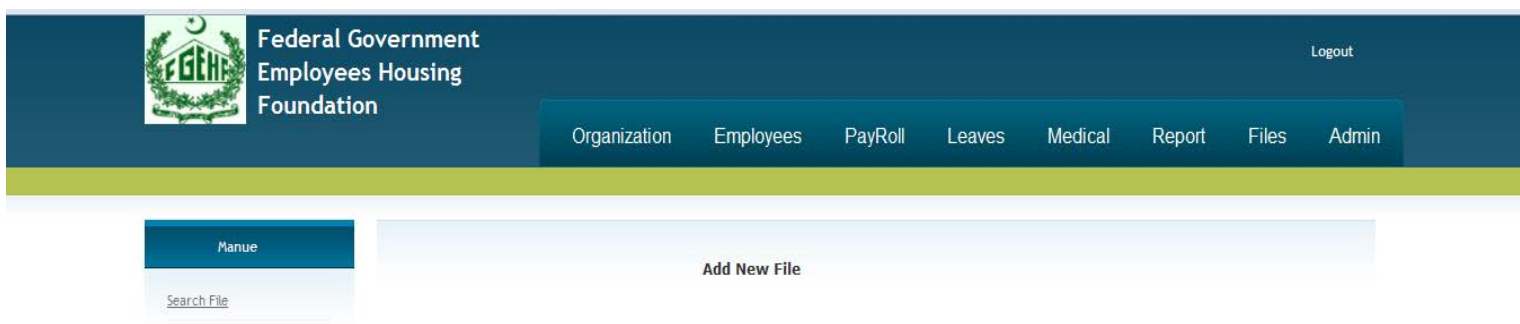

Add New File

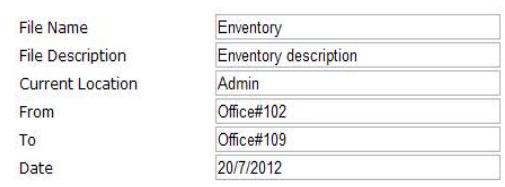

Save $\underline{\text { Cancel }}$

Figure 12. File page.

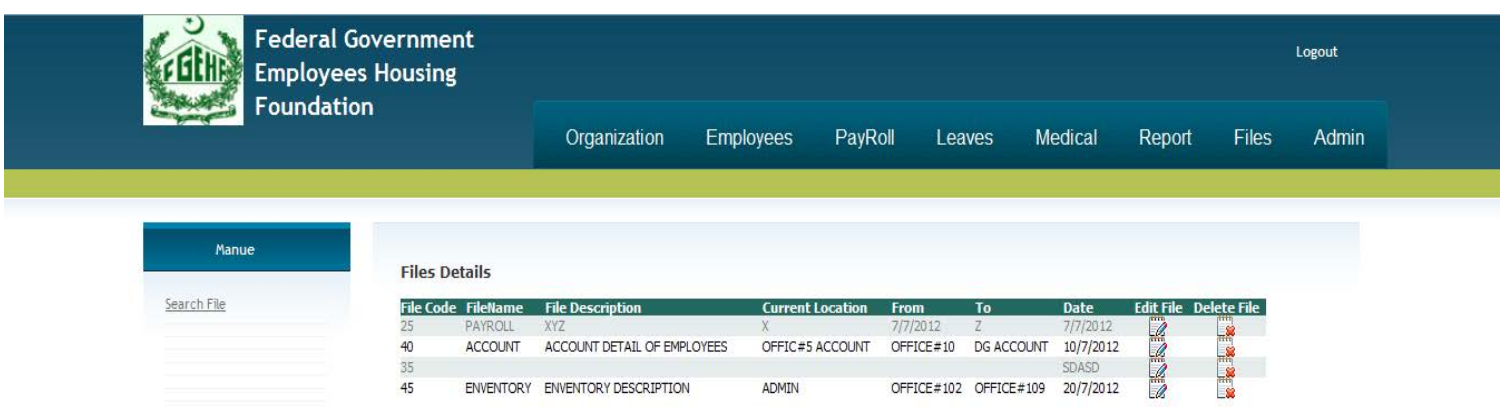

Figure 13. File view page.

users could also add new file record by clicking the add file button (Figure 13).

\subsection{Generate Employee Report page}

User can generate employee's report by clicking on the report tab. In drop-down menu multiple option are available to generate reports. If the user wants to generate report by employee CNIC number, user enters employee
CNIC\# and clicks on search button employee (Figure 14). The report is generated instantly.

\section{Conclusion and Future Enhancements}

HRMS has become an important system for HR department anywhere; however, any generic HRMS system 


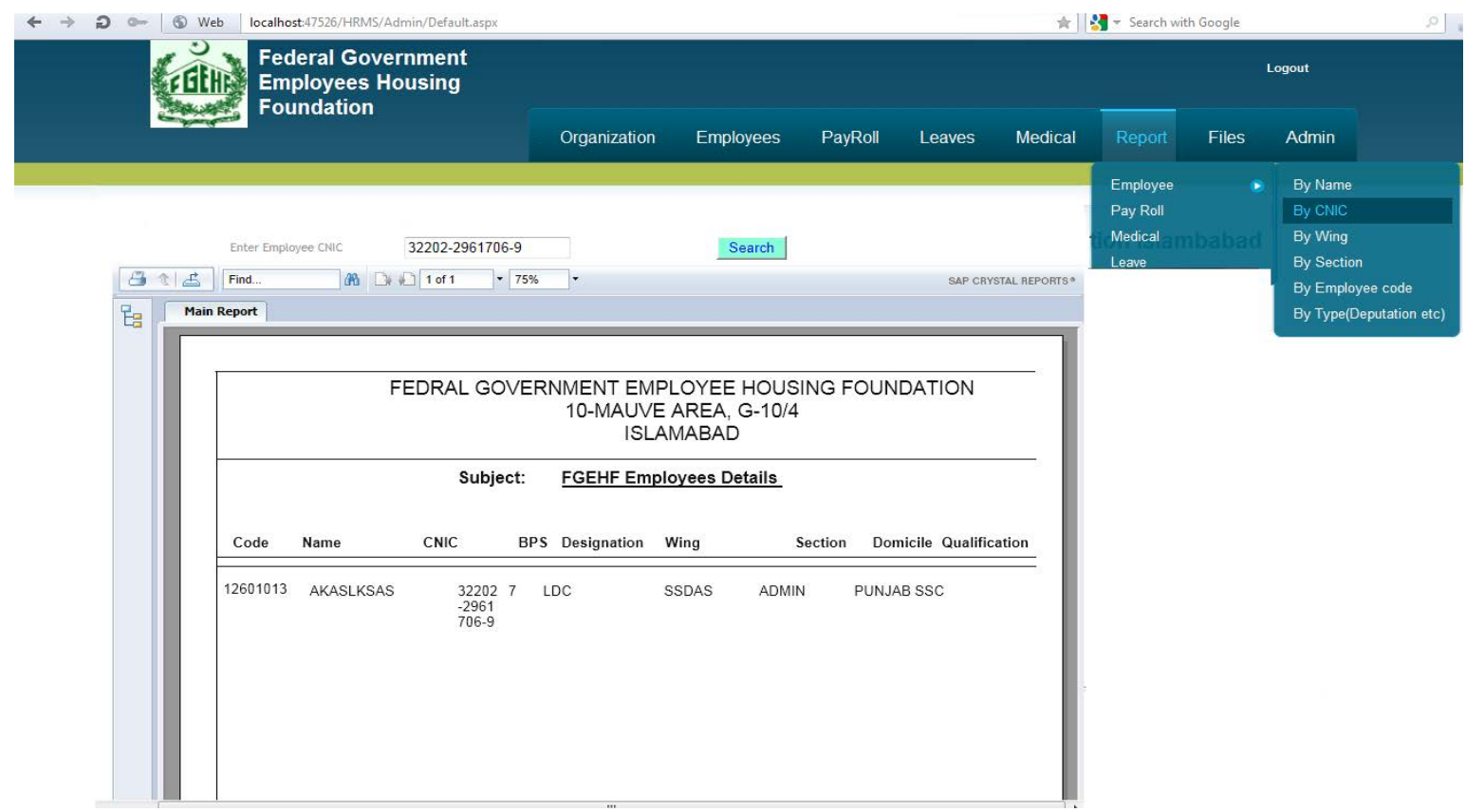

Figure 14. Employee report page.

would not be able to address fully the concerns of HR department. We developed a system to assist HR in managing the employee record in easier, effective and efficient manner. Our proposed system reduces the incurred cost and saves a lot of valuable time by avoiding any manual work. Additionally, it ensures all the data should be secure and should not be accessed by the unauthorized users. Each of the users could be assigned a particular role to access the different functionalities of the proposed system. The underlying aim of our solution is to reduce the manual workload of the administrative activities, such as maintenance and management of the file records, employee's payroll processing and other related tasks.

As a future work, we would be improving the system as there always remains the chance of improvement. We would also be extending and enhancing its features to make it more useful, reliable and user friendly.

\section{References}

1. Panhwar MA. Mobile based Healthcare Application Model for Hyderabad, Pakistan. Indian Journal of Science and Technology. 2018; 11(32):1-4. https://doi.org/10.17485/ ijst/2018/v11i32/131050

2. What is jQuery? Available from http://www.webhostdesignpost.com/website/whatisjqueryandwhy.html

3. Pressman RS. Software engineering: A practitioner's approach. 5th ed. McGraw-Hill Series in Computer Science; 2001. p. 1-860.

4. Somerville I. Software engineering. 9th ed. Addison-Wesley Publishing Company Inc; 2010.

5. Yadav M. Project report on human resource management; 2014. p. 1-86. PMCid:PMC5598519

6. Microsoft. SQL Server Management Studio (SSMS). Available from: http://msdn.microsoft.com/en-us/library/ ms174173.aspx

7. Code project. 3-tier architecture in C\#. Available from: http:// www.codeproject.com/Articles/11128/3-tier-architecture-in-C 